\title{
A Bayesian network meta-analysis on second-line systemic therapy in advanced gastric cancer
}

\author{
Xiaofu Zhu' ${ }^{1}$ Yoo-Joung $\mathrm{Ko}^{2} \cdot \mathrm{Scott}_{\mathrm{Berry}}{ }^{2} \cdot \mathrm{Keya} \mathrm{Shah}^{2} \cdot$ Esther Lee $^{2}$. \\ Kelvin Chan ${ }^{2,3,4}$
}

Received: 29 March 2016/Accepted: 2 October 2016/Published online: 8 October 2016

(c) The International Gastric Cancer Association and The Japanese Gastric Cancer Association 2016

\begin{abstract}
Background It is unclear which regimen is the most efficacious among the available therapies for advanced gastric cancer in the second-line setting. We performed a network meta-analysis to determine their relative benefits.

Methods We conducted a systematic review of randomized controlled trials (RCTs) through the MEDLINE, Embase, and Cochrane Central Register of Controlled Trials databases and American Society of Clinical Oncology abstracts up to June 2014 to identify phase III RCTs on advanced gastric cancer in the second-line setting. Overall survival (OS) data were the primary outcome of interest. Hazard ratios (HRs) were extracted from the publications on the basis of reported values or were extracted from survival curves by established methods. A Bayesian network meta-analysis was performed with WinBUGS to compare all regimens simultaneously.

Results Eight RCTs (2439 patients) were identified and contained extractable data for quantitative analysis. Network meta-analysis showed that paclitaxel plus ramucirumab was superior to single-agent ramucirumab [OS HR $0.51,95 \%$ credible region (CR) 0.30-0.86], paclitaxel (OS HR 0.81, $95 \%$ CR 0.68-0.96), docetaxel (OS HR 0.56, $95 \%$ CR 0.33-0.94), and irinotecan (OS HR 0.71, $95 \%$
\end{abstract}

Kelvin Chan

kelvin.chan@sunnybrook.ca

1 Cross Cancer Institute, Edmonton, AB T6G 1Z2, Canada

2 Sunnybrook Odette Cancer Centre, University of Toronto, Toronto, ON M4N 3M5, Canada

3 Division of Biostatistics, Dalla Lana School of Public Health, University of Toronto, Toronto, ON M5T 3M7, Canada

4 Canadian Centre for Applied Research in Cancer Control (ARCC), Toronto, Canada
CR 0.52-0.99). Paclitaxel plus ramucirumab also had an $89 \%$ probability of being the best regimen among all these regimens. Single-agent ramucirumab, paclitaxel, docetaxel, and irinotecan were comparable to each other with respect to OS and were superior to best supportive care.

Conclusions This is the first network meta-analysis to compare all second-line regimens reported in phase III gastric cancer trials. The results suggest the paclitaxel plus ramucirumab combination is the most effective therapy and should be the reference regimen for future comparative trials.

Keywords Gastric cancer · Network meta-analysis · Systemic therapy $\cdot$ Second-line therapy

\section{Introduction}

Gastric carcinoma is the fifth most common cancer diagnosed worldwide, with nearly a million new cases in 2012 [1]. In North America, most patients present with either locally advanced or metastatic disease at diagnosis, and even for patients who have localized disease and who are treated with curative-intent surgery, the recurrence rate is unacceptably high $[2,3]$. Even with modern neoadjuvant or adjuvant chemotherapy and/or radiotherapy, more than half of patients undergoing gastrectomy will experience disease recurrence $[4,5]$.

For patients with metastatic or locally advanced unresectable gastric cancer, palliative chemotherapy can offer palliation of symptoms and can prolong survival. A systematic review of chemotherapy trials in the advanced setting showed first-line chemotherapy increased survival compared with best supportive care (BSC), and combination chemotherapy was superior to single-agent regimens 
[6]. However, the duration of disease control is relatively brief, and progression-free survival or the time to progression from first-line phase III randomized controlled trials (RCTs) in the past decade has consistently been between 4 and 7 months [7-11].

Second-line systemic therapy can be considered for patients whose disease progresses with first-line therapy and who maintain a relatively good performance status. Until recently, however, treatment options have been limited. In the early part of this decade, three randomized phase III trials compared docetaxel or irinotecan against BSC [12-14]. Although limited by relatively small samples sizes, each trial revealed a statistically significant and consistent increase in overall survival by approximately 1.5 months. These results were analyzed in a meta-analysis in 2013, which showed active treatment provided a $36 \%$ reduction in the risk of death as compared with BSC [15]. This meta-analysis relied on direct pairwise comparisons, and even though it demonstrated the efficacy of active treatment against a control, it was not able to delineate the relative benefits of each treatment regimen.

Since the publication of that meta-analysis, a number of additional phase III studies have been completed showing survival benefit with other agents, expanding the therapeutic options available in the second-line setting [16-20]. Targeted agents included ramucirumab, a monoclonal antibody directed against vascular endothelial growth factor receptor 2, and everolimus, an inhibitor of the phosphatidylinositol 3-kinase-AKT-mammalian target of rapamycin pathway $[16,19]$. Additional trials compared multiagent regimens with single agents $[17,20]$.

There is currently no contemporary meta-analysis that analyzes the results from all the presently available phase III trials. The comparator arms of the different studies are variable, and it is difficult to draw conclusions from crosstrial comparisons. For example, ramucirumab has been examined in two phase III trials to date, one as monotherapy against placebo and one in combination with paclitaxel against paclitaxel alone in similar patient populations. With pairwise comparisons alone, it is not possible to determine the relative efficacy of ramucirumab alone versus other active treatments. The lack of clarity in comparative efficacy renders the selection of optimum therapy in the second-line setting difficult.

In the absence of direct head-to-head treatment comparisons, network meta-analyses can synthesize the results of RCTs by analyzing both direct and indirect evidence, thereby bridging the current gap between the various trials that do not have the same comparator arm. By constructing a network containing all available treatments and linking them via existing comparisons, one can calculate the effect of each treatment versus all other treatments simultaneously across all linkages. This method allows inclusion of a greater amount of evidence than traditional meta-analyses, which rely on only direct pairwise comparisons, thereby improving precision of estimation of effect [21, 22]. Owing to their versatility, network meta-analyses are increasingly being used to address knowledge gaps in medical sciences, including the field of oncology. Recently, network metaanalyses have explored adjuvant therapy for resected pancreatic cancer, optimal treatment of advanced colorectal cancer, and adequacy of surgical margins in ductal carcinoma in situ [23-25].

This purpose of this study was to conduct a network meta-analysis to create a network of therapeutic regimens for advanced gastric cancer in the second-line setting, and to determine their relative efficacy. The results will aid in the determination of the optimal treatment strategy, and will help guide future efforts in advancing care in this area.

\section{Materials and methods}

\section{Search strategy}

The MEDLINE, Embase, and Cochrane Central Register of Controlled Trials (CENTRAL) databases and American Society of Clinical Oncology abstracts were searched for RCTs up to June 2014 that compared systemic therapies against either other active treatments or BSC. Patients must have been treated with one or more lines of systemic therapy for advanced disease. As the chief aim of this study was to compare regimens in the second-line setting, eligible trials must have included patients who were pretreated with one prior line of therapy. Studies that included patients pretreated with one or more lines of therapy were allowed. Only prospective phase III trials reported in English were included. No limitations were placed on the type of first-line therapy; however, studies that focused solely on patients with gastric cancers overexpressing human epidermal growth factor 2 were excluded. The trials must have included patients with cancer of the stomach or gastroesophageal junction.

\section{Data extraction and analysis}

Two reviewers (X.Z. and E.L.) independently examined publications that met the inclusion criteria. Discrepancies were resolved either by consensus or through adjudication by a third reviewer (K.C.). The primary end point of this study was overall survival, which was expressed as a hazard ratio (HR). Outcomes were reported as unadjusted HRs with accompanying $95 \%$ confidence intervals (CIs). In cases where the HR and CI were not explicitly reported, they were calculated with use of the reported log-rank $p$ values and the number of reported events following 
established methods [26]. When HRs could not be extracted on the basis of the results reported in the literature, they were computed by extraction of survival curve coordinates with digitizing software (Digitizeit) with a high degree of accuracy following established methods [27].

\section{Statistical analysis}

Estimates of efficacy were calculated first from direct pairwise comparisons. Mixed treatment comparisons were generated from inclusion of indirect evidence as part of the Bayesian network.

The study by Kang et al. [13] warranted additional considerations, as the active treatment arm received a mixed treatment comprising docetaxel or irinotecan. To allow incorporation into the treatment network, docetaxel versus BSC and irinotecan versus BSC were analyzed separately.

The Bayesian network meta-analysis was performed with the Bayesian Markov chain Monte Carlo sampling in version 1.4.3 of WinBUGS. In WinBUGS, three chains were fit with 40,000 burn-ins and 40,000 iterations each. We modeled $\log (\mathrm{HR})$ with a normal distribution with a vague prior of $N(0$, tau $=0.0001)$. Noninformative priors were used because this allowed the trial data to inform the results, rather than letting strong priors dictate the results. Effect sizes for the Bayesian network meta-analysis were described with $95 \%$ credible regions (CRs), since "credible region" is a more appropriate term than "confidence interval" when one is conducting Bayesian mixed treatment comparisons in which direct evidence and indirect evidence are combined. The results generated from the network meta-analysis were compared with the results from the direct RCTs to ensure consistency.

We calculated the probability of each regimen being the best treatment by ranking the relative efficacies of all regimens in each iteration and then calculating the proportion of each regimen being ranked first across all iterations.

To translate the Bayesian network HRs into more clinically meaningful values, 6-month overall survival rates were calculated according to the methods outlined by Altman and Andersen [28, 29]. The 6-month overall survival rate from the placebo arm of the REGARD trial was used as the reference [16].

The results of these analyses were reported in accordance with the guidelines outlined by the Quality of Reporting of Meta-analyses (QUOROM) and the International Society for Pharmacoeconomics and Outcomes Research (ISPOR). For the eligible studies included in this review, assessment of risk bias was conducted by at least two authors using the Cochrane risk of bias assessment tool.

\section{Results}

\section{Literature search results}

A PRISMA diagram summarizes the literature search results and the selection process for the studies included in the network meta-analysis (Fig. 1). In total, 6942 studies were retrieved during the literature search, of which 1666 were duplicates and were removed. Of the remaining 5276 abstracts, 5163 were removed for not fulfilling the inclusion criteria. Full texts of the remaining 113 studies were reviewed, and 105 studies were excluded. Eight RCTs were obtained for the final quantitative meta-analysis $(N=2439$ patients) $[12-14,16-20]$. All trials included were randomized and followed intention-to-treat analysis for the primary end points.

The study characteristics of the publications included in the meta-analysis are shown in Table 1. The network analysis diagram is shown in Fig. 2. All trials were randomized with two comparator arms. Five trials compared cytotoxic chemotherapy or targeted therapy with $\mathrm{BSC} /$ placebo, whereas three trials had active treatments in both arms.

\section{Risk of bias assessment}

Two independent reviewers evaluated the quality of evidence reported in each study using the Cochrane risk of bias tool. Minimal bias was found among all studies included. A summary of the risk of bias for each included study is shown in Fig. 3. Since all the studies included in the network meta-analysis were randomized, selection and attrition bias were minimized. However, the method of randomization and allocation concealment were not explicitly stated in all studies, which may have potentially introduced selection bias. Except for the trial of Higuchi et al. [17], all trials had overall survival as the primary end point, which is objective and is expected to carry a low risk of detection bias.

\section{Comparison of regimens}

Of the eight trials included, five trials found statistically significant differences, including for docetaxel versus placebo /BSC (HR 0.67, $95 \%$ CI 0.49-0.92) [14], irinotecan versus placebo/BSC (HR 0.58, $95 \%$ CI $0.40-0.85$; and HR $0.48,95 \%$ CI $0.25-0.92$ ) [12, 13], ramucirumab versus placebo/BSC (HR 0.78, $95 \%$ CI 0.60-1.00) [16], and paclitaxel plus ramucirumab versus paclitaxel (HR 0.81, $95 \%$ CI 0.68-0.96) [20].

Regimen comparisons that did not produce statistically significant differences included irinotecan plus cisplatin 
Fig. 1 PRISMA flow diagram showing inclusion and exclusion of studies. ASCO American Society of Clinical Oncology, HER2 human epidermal growth factor 2

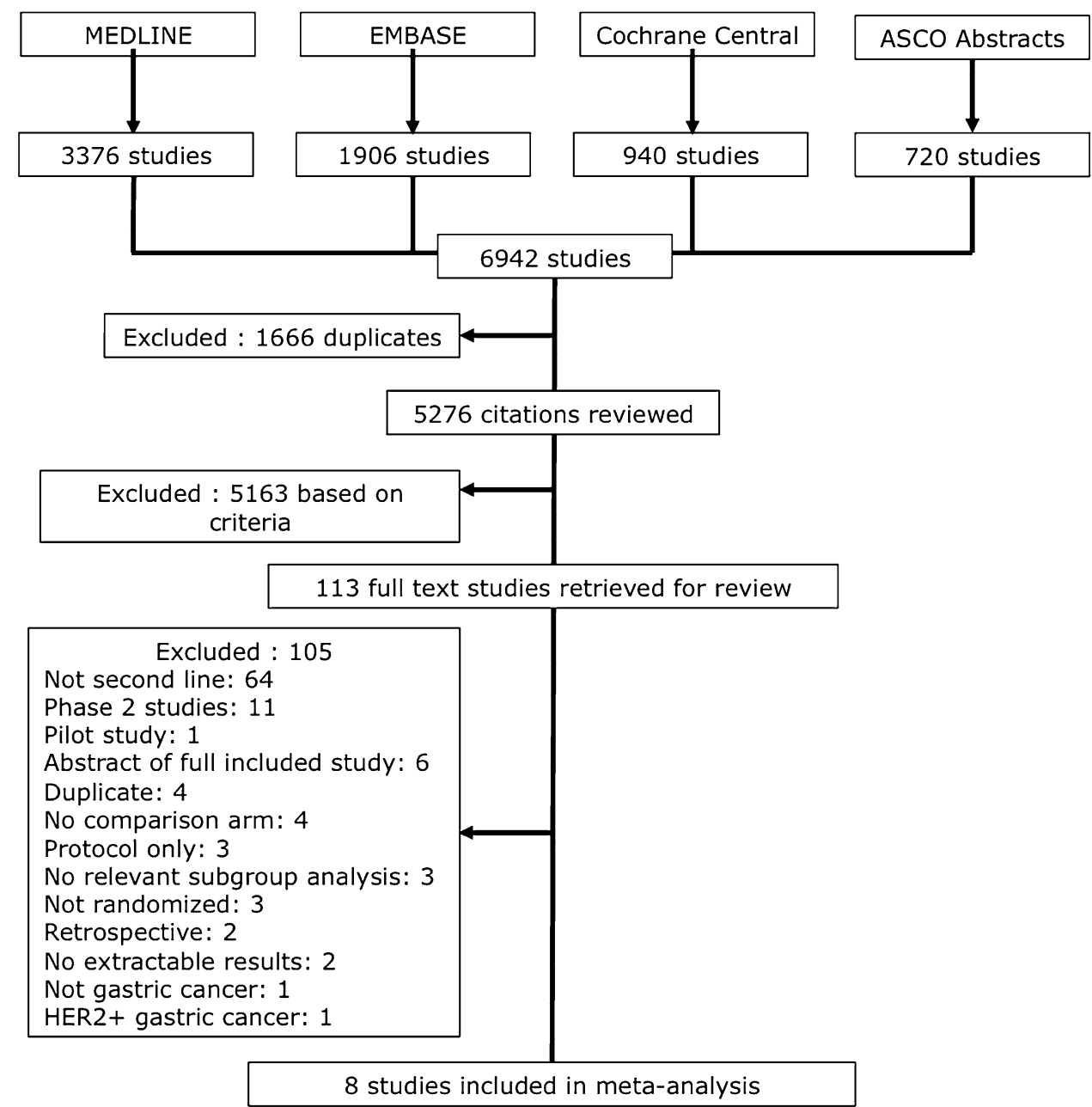

Table 1 Characteristics of eligible trials

\begin{tabular}{|c|c|c|c|c|c|c|}
\hline \multirow{2}{*}{$\frac{\text { Study }}{\text { Thuss-Patience et al. [12] }}$} & \multirow{2}{*}{$\frac{\text { Patients }}{21}$} & \multirow{2}{*}{$\begin{array}{l}\text { Treatment } \\
\text { Iri }\end{array}$} & \multicolumn{2}{|c|}{ Median OS (months) } & \multirow{2}{*}{$\frac{\text { 2nd line }(\%)}{100}$} & \multirow{2}{*}{$\frac{\text { EGJ/stomach }(\%}{100}$} \\
\hline & & & 4.0 & $p=0.012$ & & \\
\hline & 19 & BSC & 2.4 & & & \\
\hline \multirow[t]{2}{*}{ Kang et al. [13] } & $66 / 60$ & Doc or Iri & 5.3 & $p=0.007$ & 73 & 100 \\
\hline & 62 & BSC & 3.8 & & & \\
\hline \multirow[t]{2}{*}{ Ford et al. [14] } & 84 & Doc & 5.2 & $p=0.01$ & NR & 80 \\
\hline & 84 & BSC & 3.6 & & & \\
\hline \multirow[t]{2}{*}{ Hironaka et al. [18] } & 108 & $\mathrm{Pac}$ & 9.5 & $p=0.38$ & 100 & 100 \\
\hline & 111 & Iri & 8.4 & & & \\
\hline \multirow[t]{2}{*}{ Ohtsu et al. [19] } & 439 & Eve & 5.4 & $p=0.12$ & 48 & 100 \\
\hline & 217 & Pbo & 4.3 & & & \\
\hline \multirow[t]{2}{*}{ Higuchi et al. [17] } & 64 & Iri + Cis & 10.7 & $p=0.98$ & NR & 100 \\
\hline & 63 & Iri & 10.1 & & & \\
\hline \multirow[t]{2}{*}{ Fuchs et al. [16] } & 238 & Ram & 5.2 & $p=0.047$ & 100 & 100 \\
\hline & 117 & Pbo & 3.8 & & & \\
\hline \multirow[t]{2}{*}{ Wilke et al. [20] } & 330 & $\mathrm{Pac}+\mathrm{Ram}$ & 9.6 & $p=0.02$ & 100 & 100 \\
\hline & 335 & $\mathrm{Pac}$ & 7.4 & & & \\
\hline
\end{tabular}

$B S C$ best supportive care, $C i s$ cisplatin, Doc docetaxel, EGJ esophagogastric junction, Eve everolimus, Iri irinotecan, $N R$ not reported, $O S$ overall survival, $P a c$ paclitaxel, $P b o$ placebo, Ram ramucirumab 
Fig. 2 Treatment strategy network. Numbers within circles represent the total number of patients receiving each therapy within the network. Numbers between circles represent the number of studies comparing different regimens. $B S C$ best supportive care, Cis cisplatin, Doc docetaxel, Eve everolimus, Iri irinotecan, Pac paclitaxel, $P b o$ placebo, Ram ramucirumab

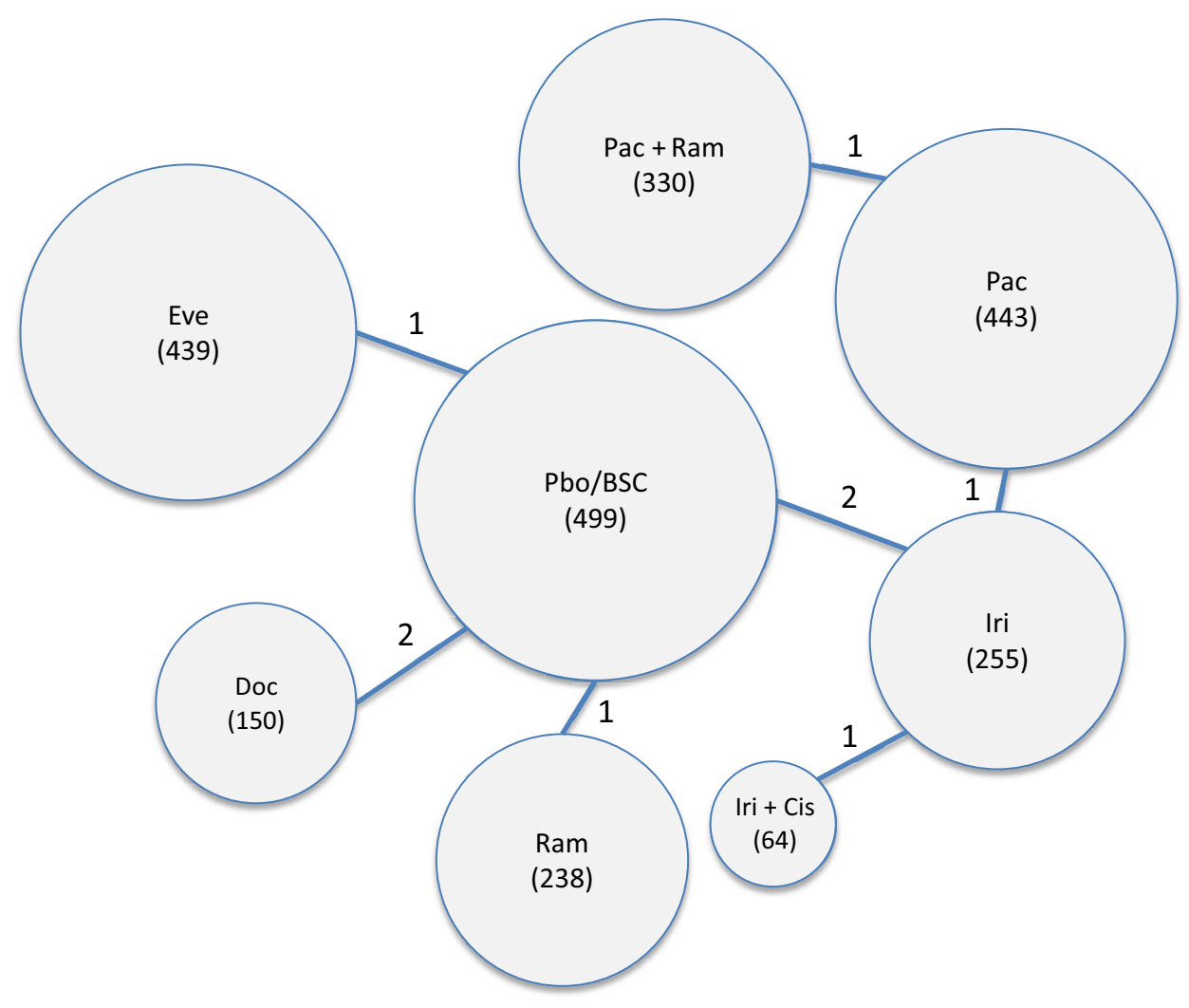

Random sequence generation (selection bias)

Allocation concealment (selection bias)

Blinding of participants and personnel (performance bias)

Blinding of outcome assessment (detection bias)

Incomplete outcome data (attrition bias)

Selective reporting (reporting bias)

Other bias

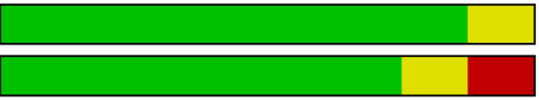

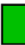

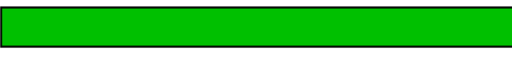

L

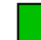

versus irinotecan (HR 1.00, $95 \%$ CI 0.69-1.44) [17], paclitaxel versus irinotecan (HR 0.89, $95 \%$ CI 0.67-1.16) [18], docetaxel versus placebo/BSC (HR 0.76, $95 \%$ CI 0.53-1.09) [13], and everolimus versus placebo/BSC (HR $0.90,95 \%$ CI $0.75-1.08$ ) [19].

Each regimen was simultaneously compared against all other regimens through the Bayesian network meta-analysis, and efficacy results are reported as HRs with $95 \%$ CRs. The results are shown in Fig. 4. Paclitaxel plus ramucirumab was superior to docetaxel (HR 0.56, $95 \%$ CR 0.33-0.94), irinotecan (HR 0.71, $95 \%$ CR 0.52-0.99), paclitaxel alone (HR 0.81, $95 \%$ CR 0.68-0.96), and ramucirumab alone (HR 0.51, 95\% CR 0.30-0.86). Paclitaxel plus ramucirumab trended toward superiority compared with irinotecan plus cisplatin (HR 0.71, $95 \%$ CR 0.44-1.17). The cytotoxic chemotherapy regimens tested were comparable against one another. All regimens except everolimus were more efficacious than placebo/ BSC.

The estimated 6-month overall survival rates are shown in Table 2. The 6-month overall survival rate with placebo in REGARD, reported as $32 \%$, was used as the control. This was chosen as this study is one of the most recently completed and published trials in the second-line advanced 
Fig. 4 Hazard ratio comparisons for overall survival from network meta-analysis comparisons. Median values are given with $95 \%$ credible regions. Hazard ratios are expressed as experimental versus control. $B S C$ best supportive care, Cis cisplatin, Doc docetaxel, Eve everolimus, Iri irinotecan, Pac paclitaxel, Pbo placebo, Ram ramucirumab

\begin{tabular}{|c|c|c|c|c|c|c|c|c|}
\hline \multirow{2}{*}{$\frac{\text { Control }}{\text { Treatment }}$} & \multicolumn{8}{|c|}{ Experimental } \\
\hline & Pbo & Doc & Iri & Iri + Cis & Pac & Pac + Ram & Ram & Eve \\
\hline Pbo & & $\begin{array}{c}0.71 \\
0.56 \cdot 0.90\end{array}$ & $\begin{array}{c}0.55 \\
0.40-0.77\end{array}$ & $\begin{array}{c}0.55 \\
0.34-0.91\end{array}$ & $\begin{array}{c}0.49 \\
0.32-0.75\end{array}$ & $\begin{array}{c}0.40 \\
0.25-0.63\end{array}$ & $\begin{array}{c}0.78 \\
0.60-1.00\end{array}$ & $\begin{array}{c}0.90 \\
0.75-1.08\end{array}$ \\
\hline Doc & $\begin{array}{c}0.78 \\
1.11-1.79\end{array}$ & & $\begin{array}{c}0.78 \\
0.52-1.17\end{array}$ & $\begin{array}{c}0.78 \\
0.45-1.35\end{array}$ & $\begin{array}{c}0.69 \\
0.42-1.13\end{array}$ & $\begin{array}{c}0.56 \\
0.33-0.94\end{array}$ & $\begin{array}{c}1.10 \\
0.78-1.55\end{array}$ & $\begin{array}{c}1.27 \\
0.94-1.71\end{array}$ \\
\hline Iri & $\begin{array}{c}1.82 \\
1.30-2.50\end{array}$ & $\begin{array}{c}1.28 \\
0.85-1.92\end{array}$ & & $\begin{array}{c}1.00 \\
0.69-1.44\end{array}$ & $\begin{array}{c}0.89 \\
0.67-1.16\end{array}$ & $\begin{array}{c}0.71 \\
0.52-0.99\end{array}$ & $\begin{array}{c}1.40 \\
0.93-2.12\end{array}$ & $\begin{array}{c}1.63 \\
1.12-2.37\end{array}$ \\
\hline Iri + Cis & $\begin{array}{c}1.82 \\
1.10-2.94\end{array}$ & $\begin{array}{c}1.28 \\
0.74-2.22\end{array}$ & $\begin{array}{c}1.00 \\
0.69-1.45\end{array}$ & & $\begin{array}{c}0.89 \\
0.56-1.40\end{array}$ & $\begin{array}{c}0.71 \\
0.44-1.17\end{array}$ & $\begin{array}{c}1.40 \\
0.81-2.44\end{array}$ & $\begin{array}{c}1.63 \\
0.96-2.76\end{array}$ \\
\hline Pac & $\begin{array}{c}2.04 \\
1.33-3.13\end{array}$ & $\begin{array}{c}1.45 \\
0.88-2.38\end{array}$ & $\begin{array}{c}1.12 \\
0.86-1.49\end{array}$ & $\begin{array}{c}1.12 \\
0.86-1.49\end{array}$ & & $\begin{array}{c}0.81 \\
0.68-0.96\end{array}$ & $\begin{array}{c}1.59 \\
0.96-2.59\end{array}$ & $\begin{array}{c}1.84 \\
1.16-2.92\end{array}$ \\
\hline Pac + Ram & $\begin{array}{c}2.50 \\
1.59-4.00\end{array}$ & $\begin{array}{c}1.79 \\
1.06-3.03\end{array}$ & $\begin{array}{c}1.41 \\
1.01-1.92\end{array}$ & $\begin{array}{c}1.41 \\
0.85-2.27\end{array}$ & $\begin{array}{c}1.23 \\
1.04-1.47\end{array}$ & & $\begin{array}{c}1.96 \\
1.16-3.32\end{array}$ & $\begin{array}{c}2.28 \\
1.39-3.73\end{array}$ \\
\hline Ram & $\begin{array}{c}1.28 \\
1.00-1.67\end{array}$ & $\begin{array}{c}0.91 \\
0.65-1.28\end{array}$ & $\begin{array}{c}0.71 \\
0.47-1.08\end{array}$ & $\begin{array}{c}0.71 \\
0.41-1.23\end{array}$ & $\begin{array}{c}0.63 \\
0.39-1.04\end{array}$ & $\begin{array}{c}0.51 \\
0.30-0.86\end{array}$ & & $\begin{array}{c}1.16 \\
0.85-1.58\end{array}$ \\
\hline Eve & $\begin{array}{c}1.11 \\
0.93-1.33\end{array}$ & $\begin{array}{c}0.79 \\
0.58-1.06\end{array}$ & $\begin{array}{c}0.61 \\
0.42-0.89\end{array}$ & $\begin{array}{c}0.61 \\
0.36-1.04\end{array}$ & $\begin{array}{c}0.54 \\
0.34-0.86\end{array}$ & $\begin{array}{c}0.44 \\
0.27-0.72\end{array}$ & $\begin{array}{c}0.86 \\
0.63-1.18\end{array}$ & \\
\hline
\end{tabular}

Table 2 Estimated 6-month overall survival rate based on hazard ratios from network meta-analysis

\begin{tabular}{lll}
\hline Regimen & Hazard ratio & 6-month OS (\%) \\
\hline Pbo/BSC & 1.00 & 32 \\
Doc & 0.71 & 45 \\
Iri & 0.55 & 53 \\
Iri + Cis & 0.55 & 53 \\
Pac & 0.49 & 57 \\
Pac + Ram & 0.40 & 63 \\
Ram & 0.78 & 41 \\
Eve & 0.90 & 36 \\
\hline
\end{tabular}

$B S C$ best supportive care, Cis cisplatin, Doc docetaxel, Eve everolimus, Iri irinotecan, $O S$ overall survival, $P a c$ paclitaxel, Pbo placebo, Ram ramucirumab

gastric cancer setting, with a large number of patients, and likely reflective of contemporary treatment practices. In our model, paclitaxel plus ramucirumab increased the 6-month overall survival rate over BSC from 32 to $63 \%$, whereas the single agents ramucirumab, paclitaxel, docetaxel, and irinotecan resulted in a 6-month overall survival rate of $41-57 \%$.

The multiple treatment comparison showed that paclitaxel plus ramucirumab had the highest probability of being the most effective regimen $(88.9 \%)$ in terms of overall survival, and irinotecan plus cisplatin was a distant second $(8.5 \%)$. The next best regimens were docetaxel $(1.0 \%)$, irinotecan $(0.9 \%)$, paclitaxel $(0.5 \%)$, and ramucirumab $(0.3 \%)$. Figure 5 shows the breakdown of probabilities.

Consistency was assessed by comparison of the HRs of the pairwise results from direct RCTs with the

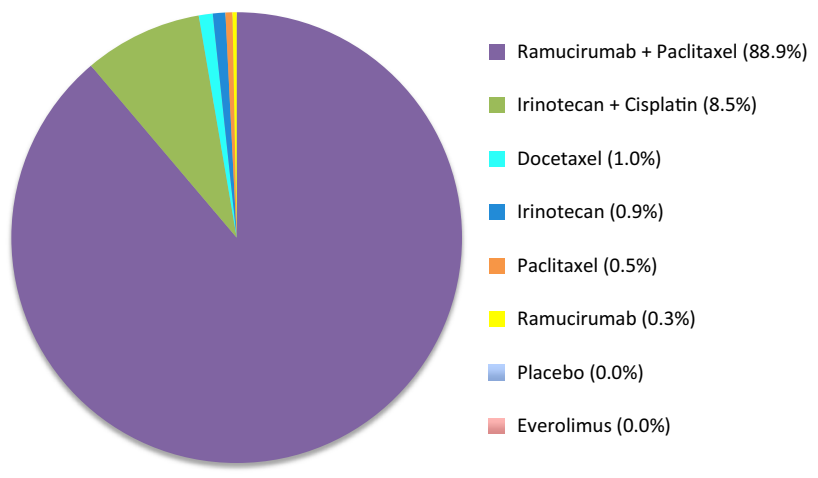

Fig. 5 Probability of each treatment being the best for overall survival

corresponding results from the network meta-analysis. The results are shown in Table 3. The results of the network meta-analysis are very similar to those seen in direct comparisons, suggesting consistency within the model.

\section{Discussion}

This network meta-analysis showed that paclitaxel plus ramucirumab was the regimen with the highest probability of being the most effective second-line regimen that has been studied in phase III trials. The RAINBOW trial, which compared paclitaxel plus ramucirumab versus paclitaxel alone, showed improvement with the addition of the targeted agent to the taxane backbone [20]. However, to date this combination has not been compared directly with any other established regimens. Through our Bayesian network meta-analysis, we were able to perform indirect 
Table 3 Results from direct pairwise comparisons and network meta-analysis (NMA) comparisons between regimens

\begin{tabular}{llll}
\hline Study & Comparison & $\begin{array}{l}\text { HR from direct pairwise } \\
\text { comparisons from RCTs }^{\mathrm{a}}\end{array}$ & HR from NMA comparisons ${ }^{\mathrm{b}}$ \\
\hline Ford et al. [14] & Doc vs Pbo/BSC & $0.67(0.49-0.92)$ & $0.71(0.56-0.90)$ \\
Fuchs et al. [16] & Ram vs Pbo/BSC & $0.78(0.60-1.00)$ & $0.78(0.60-1.00)$ \\
Higuchi et al. [17] & Iri + Cis vs Iri & $1.00(0.69-1.44)$ & $1.00(0.69-1.44)$ \\
Hironaka et al. [18] & Pac vs Iri & $0.89(0.67-1.16)$ & $0.89(0.67-1.16)$ \\
Kang et al. [11] & Doc vs Pbo/BSC & $0.76(0.53-1.09)$ & $0.71(0.56-0.90)$ \\
Kang et al. [13] & Iri vs Pbo/BSC & $0.58(0.40-0.85)$ & $0.55(0.40-0.77)$ \\
Ohtsu et al. [19] & Eve vs Pbo/BSC & $0.90(0.75-1.08)$ & $0.90(0.75-1.08)$ \\
Thuss-Patience et al. [12] & Iri vs Pbo/BSC & $0.48(0.25-0.92)$ & $0.55(0.40-0.77)$ \\
Wilke et al. [20] & Pac + Ram vs Pac & $0.81(0.68-0.96)$ & $0.81(0.68-0.96)$
\end{tabular}

$B S C$ best supportive care, Cis cisplatin, Doc docetaxel, Eve everolimus, Iri irinotecan, NMA network meta-analysis, Pac paclitaxel, $P b o$ placebo, Ram ramucirumab, RCTs randomized controlled trials

a The $95 \%$ confidence interval is given in parentheses

b The $95 \%$ credible region is given in parentheses

comparisons that showed paclitaxel plus ramucirumab was superior in terms of overall survival to single-agent ramucirumab, and other commonly used second-line regimens such as docetaxel, paclitaxel, and irinotecan. The combination of paclitaxel and ramucirumab has an $89 \%$ probability of being the most effective regimen among all the regimens.

One strength of our network meta-analysis is the ability to simultaneously analyze data from all published trials, even in the absence of direct head-to-head comparisons. A previous meta-analysis included three trials, all investigating active therapy against placebo or BSC, a common comparator arm [15]. Although active treatment was found to be superior, no information regarding the relative efficacy of each regimen to the other regimens could be deduced. This network meta-analysis is the first not only to incorporate new phase III trial data since the previous publication but also to directly and indirectly compare all regimens against one another to show the most effective treatments.

This network meta-analysis has a number of limitations. Data were collected and analyzed in aggregate on the basis of results reported from trials, rather than individual patient data. Although this meta-analysis focused on treatment in the second-line setting, not all trials enrolled second-line patients exclusively. Two trials included some patients with two prior lines of therapy [13, 19], and three others included patients with recurrent disease after adjuvant or neoadjuvant therapy $[14,16,17]$. Almost all of the trials in this study enrolled patients with stomach and gastroesophageal junction primary tumors, but one trial also allowed inclusion of tumors of the esophagus [14]. Because of the lack of availability of individual patient data, it was not possible to separately extract out subgroups of patients for analysis. Some of the studies represented in this network meta-analysis were conducted in Asia, whereas others were conducted mostly in Western nations. Variations in ethnicity of patients across the studies may introduce further heterogeneity, as differences in patient biology and regional treatment practices may ultimately have an impact on patient survival.

We focused on overall survival only and did not analyze progression-free survival between regimens. This was partly due to literature limitations, as some studies did not report progression-free survival for one or both comparator arms. Analysis of the remaining trials based on existing data would result in the creation of multiple small noninterconnected network fragments that are neither comprehensive nor informative. We feel the present study, which compares solely overall survival, remains clinically highly relevant as there are few treatment options beyond the second line in gastric cancer, and thus the advantages seen in overall survival are unlikely to be confounded by differential exposure to postprogression therapy.

Since work on this network meta-analysis began, two phase III studies on second-line systemic therapy in advanced gastric cancer have been published [30, 31] Both studies were initially published in abstract form at the Gastrointestinal Cancer Symposium of the American Society of Clinical Oncology, which was not prospectively selected as one of sources in our literature search, and as a result were not included in our network meta-analysis. The JACCRO GC-05 study by Tanabe et al. [30] compared irinotecan plus $\mathrm{S}-1$ versus irinotecan alone in patients pretreated with S-1. No significant difference in survival was seen between the arms. The TRICS study by Nishikawa et al. [31] compared irinotecan plus cisplatin versus irinotecan alone, also in patients pretreated with $\mathrm{S}-1$. Once 
again, both arms had comparable survival. The results from the latter study are in line with those from Higuchi et al. [17], where a similar comparison of irinotecan and cisplatin versus irinotecan was made, resulting in no significant difference between the arms. Given these negative results, it is unlikely the conclusions generated by our network meta-analysis would be significantly affected by the incorporation of these two studies into our model.

This network meta-analysis systemically reviewed currently available evidence for treatment of advanced gastric cancer in the second-line setting to address the knowledge gap regarding the optimal therapy for these patients. Through both direct and indirect comparisons, we demonstrated that the regimen of paclitaxel plus ramucirumab is likely to be the most efficacious therapy. Given the need to further improve the outcomes for these patients, further clinical trials are warranted, and this regimen should be the reference therapy for these trials.

Acknowledgments The Canadian Centre for Applied Research in Cancer Control (ARCC) is funded by the Canadian Cancer Society Research Institute grant 2015-703549.

\section{Compliance with ethical standards}

Conflict of interest Yoo-Joung Ko has received honoraria from Roche and Bristol-Myers Squibb. Scott Berry has received honoraria from Roche, Amgen, Bristol-Myers Squibb, and Sanofi. The other authors declare that they have no conflict of interest.

Ethical standards All procedures followed were in accordance with the ethical standards of the responsible committee on human experimentation (institutional and national) and with the Helsinki Declaration of 1964 and later versions. Informed consent or substitute for it was obtained from all patients for their being included in the study.

\section{References}

1. Internation Agency for Research on Cancer. GLOBOCAN cancer fact sheets: stomach cancers. 2012. Accessed 31 May 2015. http://globocan.iarc.fr/old/FactSheets/cancers/stomach-new.asp.

2. Bang Y-J, Kim Y-W, Yang H-K, Chung HC, Park Y-K, Lee KH, et al. Adjuvant capecitabine and oxaliplatin for gastric cancer after D2 gastrectomy (CLASSIC): a phase 3 open-label, randomised controlled trial. Lancet. 2012;379:315-21.

3. Sasson AR. Localized gastric cancer: chemoradiation is not always needed. Gastrointest Cancer Res. 2009;3:S22-5.

4. Cohen DJ, Leichman L. Controversies in the treatment of local and locally advanced gastric and esophageal cancers. J Clin Oncol. 2015;33:1754-9.

5. Ychou M, Boige V, Pignon J-P, Conroy T, Bouché O, Lebreton $\mathrm{G}$, et al. Perioperative chemotherapy compared with surgery alone for resectable gastroesophageal adenocarcinoma: an FNCLCC and FFCD multicenter phase III trial. J Clin Oncol. 2011;29:1715-21.

6. Wagner AD, Unverzagt S, Grothe W, Kleber G, Grothey A, Haerting J, et al. Chemotherapy for advanced gastric cancer. Cochrane Database Syst Rev. 2010;CD004064.
7. Cunningham D, Starling N, Rao S, Iveson T, Nicolson M, Coxon $\mathrm{F}$, et al. Capecitabine and oxaliplatin for advanced esophagogastric cancer. N Engl J Med. 2008;358:36-46.

8. Van Cutsem E, Moiseyenko VM, Tjulandin S, Majlis A, Constenla M, Boni C, et al. Phase III study of docetaxel and cisplatin plus fluorouracil compared with cisplatin and fluorouracil as firstline therapy for advanced gastric cancer: a report of the V325 Study Group. J Clin Oncol. 2006;24:4991-7.

9. Guimbaud R, Louvet C, Ries P, Ychou M, Maillard E, André T, et al. Prospective, randomized, multicenter, phase III study of fluorouracil, leucovorin, and irinotecan versus epirubicin, cisplatin, and capecitabine in advanced gastric adenocarcinoma: a French intergroup (Fédération Francophone de Cancérologie Digestive, Fédération Nationale des Centres de Lutte Contre le Cancer, and Groupe Coopérateur Multidisciplinaire en Oncologie) study. J Clin Oncol. 2014;32:3520-6.

10. Al-Batran S-E, Hartmann JT, Probst S, Schmalenberg H, Hollerbach S, Hofheinz R, et al. Phase III trial in metastatic gastroesophageal adenocarcinoma with fluorouracil, leucovorin plus either oxaliplatin or cisplatin: a study of the Arbeitsgemeinschaft Internistische Onkologie. J Clin Oncol. 2008;26:1435-42.

11. Kang Y-K, Kang W-K, Shin D-B, Chen J, Xiong J, Wang J, et al. Capecitabine/cisplatin versus 5-fluorouracil/cisplatin as first-line therapy in patients with advanced gastric cancer: a randomised phase III noninferiority trial. Ann Oncol. 2009;20:666-73.

12. Thuss-Patience PC, Kretzschmar A, Bichev D, Deist T, Hinke A, Breithaupt K, et al. Survival advantage for irinotecan versus best supportive care as second-line chemotherapy in gastric cancer-a randomised phase III study of the Arbeitsgemeinschaft Internistische Onkologie (AIO). Eur J Cancer. 2011;47:2306-14.

13. Kang JH, Lee SI, Lim DH, Park K-W, Oh SY, Kwon H-C, et al. Salvage chemotherapy for pretreated gastric cancer: a randomized phase III trial comparing chemotherapy plus best supportive care with best supportive care alone. J Clin Oncol. 2012;30:1513-8.

14. Ford HER, Marshall A, Bridgewater JA, Janowitz T, Coxon FY, Wadsley J, et al. Docetaxel versus active symptom control for refractory oesophagogastric adenocarcinoma (COUGAR-02): an open-label, phase 3 randomised controlled trial. Lancet Oncol. 2014;15:78-86.

15. Kim HS, Kim HJ, Kim SY, Kim TY, Lee KW, Baek SK, et al. Second-line chemotherapy versus supportive cancer treatment in advanced gastric cancer: a meta-analysis. Ann Oncol. 2013;24:2850-4.

16. Fuchs CS, Tomasek J, Yong CJ, Dumitru F, Passalacqua R, Goswami C, et al. Ramucirumab monotherapy for previously treated advanced gastric or gastro-oesophageal junction adenocarcinoma (REGARD): an international, randomised, multicentre, placebo-controlled, phase 3 trial. Lancet. 2014;383:31-9.

17. Higuchi K, Tanabe S, Shimada K, Hosaka H, Sasaki E, Nakayama $\mathrm{N}$, et al. Biweekly irinotecan plus cisplatin versus irinotecan alone as second-line treatment for advanced gastric cancer: a randomised phase III trial (TCOG GI-0801/BIRIP trial). Eur J Cancer. 2014;50:1437-45.

18. Hironaka S, Ueda S, Yasui H, Nishina T, Tsuda M, Tsumura T, et al. Randomized, open-label, phase III study comparing irinotecan with paclitaxel in patients with advanced gastric cancer without severe peritoneal metastasis after failure of prior combination chemotherapy using fluoropyrimidine plus platinum: WJOG 4007 trial. J Clin Oncol. 2013;31:4438-44.

19. Ohtsu A, Ajani JA, Bai Y-X, Bang Y-J, Chung H-C, Pan H-M, et al. Everolimus for previously treated advanced gastric cancer: results of the randomized, double-blind, phase III GRANITE-1 study. J Clin Oncol. 2013;31:3935-43. 
20. Wilke H, Muro K, Van Cutsem E, Oh S-C, Bodoky G, Shimada $\mathrm{Y}$, et al. Ramucirumab plus paclitaxel versus placebo plus paclitaxel in patients with previously treated advanced gastric or gastro-oesophageal junction adenocarcinoma (RAINBOW): a double-blind, randomised phase 3 trial. Lancet Oncol. 2014;15:1224-35.

21. Lu G, Ades AE. Combination of direct and indirect evidence in mixed treatment comparisons. Stat Med. 2004;23:3105-24.

22. Salanti G, Ades AE, Ioannidis JPA. Graphical methods and numerical summaries for presenting results from multiple-treatment meta-analysis: an overview and tutorial. J Clin Epidemiol. 2011;64:163-71.

23. Liao W-C, Chien K-L, Lin Y-L, Wu M-S, Lin J-T, Wang H-P, et al. Adjuvant treatments for resected pancreatic adenocarcinoma: a systematic review and network meta-analysis. Lancet Oncol. 2013;14:1095-103.

24. Golfinopoulos V, Salanti G, Pavlidis N, Ioannidis JPA. Survival and disease-progression benefits with treatment regimens for advanced colorectal cancer: a meta-analysis. Lancet Oncol. 2007;8:898-911.

25. Wang S-Y, Chu H, Shamliyan T, Jalal H, Kuntz KM, Kane RL, et al. Network meta-analysis of margin threshold for women with ductal carcinoma in situ. J Natl Cancer Inst. 2012;104:507-16.
26. Parmar MK, Torri V, Stewart L. Extracting summary statistics to perform meta-analyses of the published literature for survival endpoints. Stat Med. 1998;17:2815-34.

27. Guyot P, Ades AE, Ouwens MJNM, Welton NJ. Enhanced secondary analysis of survival data: reconstructing the data from published Kaplan-Meier survival curves. BMC Med Res Methodol. 2012;12:9.

28. Altman DG. Confidence intervals for the number needed to treat. BMJ. 1998;317:1309-12.

29. Altman DG, Andersen PK. Calculating the number needed to treat for trials where the outcome is time to an event. BMJ. 1999;319:1492-5.

30. Tanabe K, Fujii M, Nishikawa K, Kunisaki C, Tsuji A, Matsuhashi N, et al. Phase II/III study of second-line chemotherapy comparing irinotecan-alone with S-1 plus irinotecan in advanced gastric cancer refractory to first-line treatment with S-1 (JACCRO GC-05). Ann Oncol. 2015;26:1916-22.

31. Nishikawa K, Fujitani K, Inagaki H, Akamaru Y, Tokunaga S, Takagi $\mathrm{M}$, et al. Randomised phase III trial of second-line irinotecan plus cisplatin versus irinotecan alone in patients with advanced gastric cancer refractory to S-1 monotherapy: TRICS trial. Eur J Cancer. 2015;51:808-16. 